\title{
Prévalence de la brucellose, chlamydiose et toxoplasmose chez les petits ruminants au Mali : résultats d'une enquête séro-épidemiologique
}

\section{Prevalence of brucellosis, chlamydiosis and toxoplasmosis in small ruminants in Mali: results of an sero-epidemiological survey}

Sidibe $\mathrm{S}^{1}$, Coulibaly $\mathrm{KW}^{1}$, Sery $\mathrm{A}^{1}$, Fofana $\mathrm{M}^{1}$, Sidibe $\mathrm{F}^{2}$, Kanoute $\mathrm{M}^{2}$

1. Laboratoire Central Vétérinaire, BP. 2295, Bamako, MALI.

2. Institut Polytechnique Rural de Katibougou, BP. 06 Koulikoro,

Auteur correspondant: sidibsatigui@gmail.com

\section{Résumé}

$\mathrm{Au}$ Mali, l'élevage des espèces à cycle court en général et celui des petits ruminants en particulier constitue une source précieuse de revenus et de protéines pour les populations. Cependant, au nombre des contraintes à l'amélioration de la productivité de cet élevage, figurent les pertes en reproduction, dont les causes demeurent mal connues. Les données relatives au rôle de certaines infections microbiennes comme la brucellose à Brucella ovis, chlamydiose et la toxoplasmose sont quasi inexistantes. L'objet de la présente étude a été d'évaluer la prévalence sérologique de ces pathologies, susceptibles de causer des pertes en reproduction chez ces espèces animales. Elle a couvert quatre sites dont Kéniébougou (Cercle de Ségou), Nioro (Cercle de Nioro), Bougouni (Cercle de Bougouni) et Briga (Cercle de Koro), choisis en collaboration étroite avec les services techniques d'encadrement et éleveurs de petits ruminants desdites localités. Au total 872 sérums (368 ovins et 504 caprins) issus des sujets présentant des signes de perte en reproduction ont été testés par la méthode Elisa indirecte. Parmi ceux-ci, $81(9,28 \%)$ se sont révélés positifs $(1,26 \%$ en brucellose à Brucella ovis, $3,55 \%$ en chlamydiose à Chlamydia abortus et $4,47 \%$ en toxoplasmose à Toxoplasma gondii). Les anticorps mis en évidence sont considérés comme d'origine infectieuse, les animaux n'ayant jamais été vaccinés contre les maladies investiguées, ce qui confirme la circulation des agents étiologiques des trois infections abortives parmi les populations de petits ruminants au Mali.

Mots Clés : Enquête, Sérologie, Brucellose, Chlamydiose, Toxoplasmose, Petits ruminants, Mali.

\section{Abstract}

In Mali, the short cycle animal species livestock in general and that of small ruminants in particular is a valuable source of income and protein for the populations. However, among the constraints to the improvement of the productivity of this livestock, the losses in reproduction, whose causes remain poorly known. Data on the role of microbial infections are fragmentary. The purpose of this study was to assess the seroprevalence of brucellosis due to Brucella ovis, chlamydiosis due to Chlamydia abortus, and toxoplasmosis to Toxoplasma gondii, which may cause reproductive losses in these animal species. The study was carried in for sites in total including Keniebougou ( Segou areas), Nioro (Nioro areas), Bougouni (Bougouni areas) and Briga (Koro areas) chosen in close collaboration with the technical services et animal holders in the targeted localities. A total of 872 sera (368 sheep and 504 goats) from animals showing signs of reproductive loss were tested by the indirect Elisa method. Of these, 81 (9.28\%) were positive (1.26\% for brucellosis due to Brucella ovis, $3.55 \%$ for Chlamydia abortus chlamydiosis, and $4.47 \%$ for Toxoplasma gondii toxoplasmosis). The detected antibodies are considered to be of infectious origin, since the selected animals have never been vaccinated against the investigated diseases and this confirm the circulation of the etiological agents of the tree abortive diseases among small ruminant populations in Mali.

Key Words: Survey, Serology, Brucellosis, Chlamydiosis, Toxoplasmosis, Small ruminants, Mali

\section{Introduction}

Le sous-secteur de l'élevage occupe une place de choix dans l'économie du Mali. Il constitue la principale source de subsistance pour plus de $30 \%$ de la population malienne et contribue pour 13,6
$\%$ au PIB, 24\% à la production du secteur rural, $80 \%$ environ aux revenus des populations rurales et près de $20 \%$ aux recettes d'exportation (1).Le cheptel national occupe le premier rang dans l'espace de l'Union Economique et 
Monétaire Ouest-Africaine (UEMOA) et le second dans l'espace de la Communauté Economique des Etats de l'Afrique de l'Ouest (CEDEAO). Les effectifs de petits ruminants sont estimés 41423800 dont 17400000 ovins et 24023800 caprins (2). Malgré l'importance numérique et socio-économique des petits ruminants au Mali, au nombre des contraintes à l'amélioration de la productivité de ces espèces, figurent entre autres les nombreux cas de pertes en reproduction (avortements, infertilité, orchite, chétivité). Ces pertes en reproduction peuvent avoir des causes diverses dont certaines maladies abortives zoonotiques comme la brucellose, la chlamydiose et la toxoplasmose $(3,4,5,6$, $7,8,9,10,11,12,13,15)$. Ces maladies ont un caractère zoonotique et demeurent négligées (absence de dépistage systématique et de stratégies adaptées de lutte, diagnostic différentiel délicat). Par conséquent, les données disponibles sur la question au Mali et dans la sous- région sont parcellaires (prévalences réelles mal connues). L'objet de la présente étude a été de déterminer les prévalences sérologiques de trois de ces maladies abortives zoonotiques (brucellose, chlamydiose et toxoplasmose) chez les petits ruminants au Mali

\section{Matériel et Méthodes}

\subsection{Période et type d'étude}

Il s'agit d'une étude exploratoire à visée descriptive, menée pendant la période 2006-2010.

\subsection{Sites et matériel d'étude}

L'étude a couvert quatre sites au total dont Kéniébougou (Cercle de Ségou), Nioro (Cercle de Nioro), Bougouni (Cercle de Bougouni) et Briga (Cercle de Koro). Elle a concerné un effectif total de 7592 sujets dont 2709 à Ségou, 3166 à Nioro, 872 à Bougouni et 845 à Koro. Le matériel animal était constitué de brebis appartenant aux races «Djallonké», « Maure», «Peulh» et «Bali Bali» et de métisses issus de croisements entre les diverses races ovines. Les caprins étaient des
" Chèvres du Sahel », "Chèvres naines" (Djallonké) et métisses issus de différents croisements.La sélection des sites a été opérée en collaboration étroite avec les structures techniques régionales et locales d'encadrement et éleveurs en fonction des informations disponibles sur l'importance des effectifs de petits ruminants, l'incidence des cas de pertes en reproduction, l'accessibilité du site en toute saison, le degré de réceptivité des éleveurs, la prédominance du mode d'élevage sédentaire. Ainsi, un (01) site avait été choisi dans chacun des cercles proposés dans les localités ciblées pour abriter l'étude (4 sites au total). Situé dans le Cercle de Ségou, le site de Kéniébougou a un climat sahélien avec des hauteurs de pluies variant entre 400 et 800 $\mathrm{mm}$. La végétation est composée d'arbustes et d'épineux et la savane est arborée. Le site de Bougouni est situé en zone soudanienne et la pluviométrie y varie entre 800 et $1110 \mathrm{~mm}$ par an. La végétation est celle de la savane et le vent dominant est l'harmattan. Celui de Nioro se trouve en zone sahélienne où la pluviométrie varie entre 500 et $700 \mathrm{mn}$ par an. Il souffle principalement l'harmattan et la mousson; la végétation est composée essentiellement d'acacias et d'épineux.La localité de Koro est située en zone sahélienne chaude et sèche. La pluviométrie varie entre 350 et $500 \mathrm{~mm}$ par an. La végétation est composée de baobab, de cail-cédrat et d'épineux.Dans les différents sites de l'étude, les animaux sont généralement en divagation pendant la saison sèche, pâturent aux alentours des villages pendant la journée et rejoignent les concessions le soir. Par contre, pendant l'hivernage, ils sont en stabulation afin de protéger les cultures (enclos, cases). Les sujets des troupeaux de petite taille sont attachés aux piquets pendant la journée dans les pâturages disponibles et sont conduits le soir dans les enclos ou cases situées dans les familles. La spécificité de la zone sahélienne réside dans le fait que 
les agro-éleveurs ne construisent pas d'enclos ou de cases pour les animaux dans la majorité des cas. Ces derniers sont souvent attachés aux piquets dans la cour de la concession ou sont le plus souvent conduits par troupeaux collectifs par des bergers.

\subsection{Choix, suivi des élevages et sujets, collecte de prélèvements}

Tout le cheptel de petits ruminants des quatre sites a fait l'objet de suivi. Dans chaque cas de perte en reproduction, les animaux étaient identifiés et examinés (thermométrie, recherche d'autres signes cliniques comme le jetage, la toux, la salivation, la diarrhée) avant la collecte de prélèvements de sang. Ils ont été classés en trois groupes d'âge: 0-12 mois, 13 mois 48 mois et plus de 48 mois. Le suivi clinico-épidémiologique des troupeaux a duré deux années au cours desquelles, les données clinico-épidémiologiques ont été enregistrées à travers le recensement systématique de tous les cas de perte en reproduction (avortements, mortalités néonatales, infertilité, orchites et chétivité) et à la collecte de sang chez les animaux concernés.Le sang a été prélevé à la veine jugulaire à l'aide de tubes vacutainer sous vide et stériles. Les sérums ont été récoltés le même jour, conservés au frais jusqu'à leur expédition au Laboratoire Central Vétérinaire sous glace pour des fins d'analyse.Dans chaque cas de prélèvement, des fiches de renseignement étaient dûment remplies sur lesquelles les informations relatives à l'espèce de petits ruminants, à la race, au sexe, à l'âge et au rang de portée pour les femelles étaient notées.

\subsection{Méthodes d'analyse de laboratoire}

Les travaux de diagnostic ont été réalisés au sein de l'unité de séro-immunologie du Laboratoire Central Vétérinaire. Pour tester les sérums, des kits d'ELISA indirecte fournis par les sociétés IDVET (Montpellier, France) pour la chlamydiose à Chlamydia abortus, la toxoplasmose à Toxoplasma gondii et IDEXX (Laboratories B.V., Nederland) pour la brucellose à Brucella ovis, ont été utilisés.Les tests ont été réalisés conformément aux recommandations des fabricants. La lecture des plaques a été faite à l'aide du Lecteur ELISA (Titertech Multiscan Plus) à $450 \mathrm{~nm}$. Pour la chlamydiose et la toxoplasmose, le test était validé lorsque la valeur moyenne de la densité optique des contrôles positifs était supérieure à 0,350 et que le rapport entre la moyenne des densités optiques des contrôles positifs (DOcp) et celles des contrôles négatifs $(\mathrm{DO} \mathrm{cn})$ était supérieur à 3. Pour la chlamydiose et la toxoplasmose, les critères de séropositivité ont été déterminés à partir du seuil de positivité $(\mathrm{S} / \mathrm{P})$ qui a été calculé pour chaque échantillon selon la formule: $\mathrm{S} / \mathrm{P}=\mathrm{DO}$ échantillon/DOcp X 100.

Ainsi, les échantillons ayant donné un $\mathrm{S} / \mathrm{P}$ : - Inférieur ou égal à $40 \%$ sont considérés comme négatifs.

- Supérieur à $40 \%$ et inférieur ou égal à $50 \%$ sont considérés comme douteux

-Supérieur à $50 \%$ et inférieur ou égal à $80 \%$ sont considérés comme positifs

-Supérieur à $80 \%$ sont considérés comme fortement positifs

Pour la brucellose, le test était validé si :

- La DO du contrôle négatif ne dépasse pas 0,5 .

- La DO du contrôle positif ne dépasse pas 2,0 .

- La différence entre le contrôle négatif et positif est de $>$ ou égale à 0,3 .

Les critères de séropositivité ont été déterminés à partir de la densité optique de l'échantillon qui a été calculée selon la formule :

DO échantillon $(\%)=$ DOéch - DOneg / ODpos - DOneg X 100

Si la valeur de la densité optique de l'échantillon est :

- Inférieure à $10 \%$, le résultat est négatif.

- Supérieure ou égale à $10 \%$ et inférieur à $50 \%$, le résultat est douteux.

- Supérieure ou égale à $50 \%$, le résultat est positif.

\subsection{Analyses statistiques}


Pour vérifier l'existence de liens statistiques entre les taux de prévalences et chacun des facteurs comme le site, l'espèce de petits ruminants, la nature de la perte en reproduction et l'âge des animaux testés, le test de Khi-2 (Pearson) a été utilisé. La différence a été considérée comme significative lorsque le p-value (taux de signification) était inférieur à 0,05 .

2.6. Considérations éthiques: En prélude au démarrage des activités de suivi clinicoépidémiologique des élevages des localités choisies, des réunions d'échanges et d'informations ont été organisées avec les services techniques d'encadrement et éleveurs des localités ciblées, afin d'expliquer les objectifs de l'étude et d'obtenir leur adhésion et le consentement libre et éclairé de ces derniers. Aussi, les résultats de l'étude leur ont été restitués et des conseils pratiques ont été prodigués en vue de mieux contrôler les pathologies identifiées et de prévenir les cas de contaminations humaines éventuelles.

\section{Résultats}

\subsection{Données clinico-épidémiologiques}

Au cours de cette étude, 872 sérums ont été collectés chez 368 ovins et 504 caprins qui avaient montré des signes de perte en reproduction. La réalisation des examens sérologiques a permis d'établir des taux de prévalence sérologique de $1,26 \%$ pour la brucellose à Brucella ovis, 3,55\% pour la chlamydiose et $4,47 \%$ pour la toxoplasmose. Parmi les pertes en reproduction observées au cours de cette étude, les avortements ont occupé une place de choix (762 cas d'avortement sur 872 cas de pertes recensés). Ceci représente un taux de $87,38 \%$. La plupart des cas de pertes en reproduction ont été observés chez les caprins (57,79\%).

\subsection{Prévalence des maladies abortives étudiées dans les différents sites}

L'étude a permis d'obtenir des prévalences de $4,47 \%$ pour la toxoplasmose, $3,55 \%$ pour la chlamydiose et $1,26 \%$ pour la brucellose. L'analyse statistique a montré que ces taux ont varié en fonction des sites $(\mathrm{P}=2,2>0,05)$ (tableau I.). L'examen de ce tableau montre que les taux d'infection en brucellose à Brucella ovis est plus élevé à Koro avec un taux de 3,35\%, suivi de Bougouni (1,46\%). Par contre, l'infection est très faible voire presque inexistante à Ségou $(0,65 \%)$ et aucun cas n'a été enregistré à Nioro. Pour la chlamydiose, le site de Nioro a enregistré le taux de séroprévalence le plus élevé (10,98\%), suivi de Ségou $(1,96 \%)$ et de Bougouni $(1,95 \%)$. Le taux le plus faible pour cette infection a été mis en évidence à Koro $(0,55 \%)$ (Tableau I). En toxoplasmose, des cas n'ont été enregistrés que dans le seul site de Bougouni $(19,02 \%)$ (Tableau I).

\subsection{Séroprévalence en fonction des espèces de petits ruminants}

Pour la toxoplasmose, les analyses statistiques ont montré une différence significative au niveau des prévalences pour les deux espèces de petits ruminants. Les ovins ont enregistré le taux le plus élevé avec 7,06 \%, tandis que les caprins n'ont enregistré que $2,57 \%$. Pour la chlamydiose et la brucellose, aucune différence statistiquement significative n'a été trouvée pour les prévalences chez les deux espèces $(p=0,599$ et $p=0,253$ $>0,005)$ respectivement.

\subsection{Résultats sérologiques par groupe d'âge}

L'analyse des résultats de séroprévalence par groupe d'âge montre que les 3 maladies abortives ont été enregistrées chez les petits ruminants appartenant à tous les groupes d'âge avec des taux de prévalence plus importants pour les sujets âgés de plus de 4 ans $(5,97 \%$ en chlamydiose et $11,94 \%$ en toxoplasmose). En revanche, des cas positifs de brucellose n'ont été identifiés que chez des sujets de moins d'un an d'âge $(3,73 \%)$ et ceux issus de la tranche d'âge de 1 à 4 ans (Tableau III). Pour la brucellose et la toxoplasmose, les analyses statistiques ont mis en évidence une différence statistique 
significative entre les groupes tranches d'âge $(p=0,03$ et $p=0,00<0,05)$. Pour la chlamydiose, la différence n'est pas significative ( $p=0,07>0,05)$.

\subsection{Prévalence des maladies abortives en fonction de la nature de la perte en reproduction}

$\mathrm{Au}$ cours de cette étude, 872 cas de pertes en reproduction ont été recensés dont 762 cas d'avortements $(87,38 \%), 72$ cas de chétivité $(8,25 \%), 29$ cas d'infertilité $(3,32$ $\%), 7$ cas d'orchite $(0,80 \%)$ et 2 cas de rétention placentaire $(0,22 \%)$ (Tableau III). Les résultats obtenus montrent que parmi les 762 sujets ayant avorté, 28 étaient positifs à la chlamydiose $(3,67 \%)$, 10 à la brucellose à Brucella ovis $(1,31 \%)$ et $35(4,59 \%)$ à la toxoplasmose (Tableau IV).Aucune différence statistiquement significative n'a été établie entre les séroprévalences des 3 pathologies et la nature des pertes en reproduction $(p=0,111>0,05)$.

\section{Discussion}

La technique de l'ELISA indirecte a été retenue comme méthode de diagnostic de laboratoire en raison de sa sensibilité élevée, de sa rapidité et de sa commodité pour les sondages épidémiologiques en lieu et place de techniques de diagnostic conventionnelles comme le test de fixation du complément (14). La présente étude a permis d'établir des taux de prévalence sérologique de $3,55 \%$ pour la chlamydiose , 1,26\% pour la brucellose à Brucella ovis et $4,47 \%$ pour la toxoplasmose. Les résultats obtenus ont montré la présence des anticorps spécifiques contre Chlamydia abortus, Brucella ovis et Toxoplama gondii chez les petits ruminants. Les anticorps détectés sont d'origine infectieuse, les animaux n'ayant jamais été vaccinés contre ces maladies.La présente étude a permis d'obtenir un taux de prévalence global de $3,55 \%$ en chlamydiose qui a varié en fonction des sites $(p<0,05)$. Ce taux a été plus important dans le site de Nioro., ce qui pourrait s'expliquer par la particularité du mode d'élevage de ces espèces dans ce site (conduite groupée de troupeaux appartenant à plusieurs éleveurs du même village). Ces facteurs favorisent la transmission rapide de l'infection. Il est plus faible que celui obtenu au Sénégal avec $6,75 \%$ chez les ovins et de 19,38\% chez les caprins (4) et ceux obtenus en Tunisie $(6,04 \%)$ (11 et 12$)$ et en Mauritanie avec 15 et $30 \%$ chez les caprins et ovins respectivement (7).Aussi, la présente étude a obtenu un taux de 1,26\% pour la brucellose à Brucella ovis (agent étiologique de l'épididymite infectieuse du bélier) chez les ovins uniquement. Ce taux a varié en fonction des sites. Une prévalence globale de $4,47 \%$ a été obtenue pour la toxoplasmose et cette maladie n'a été enregistrée qu'au sein du seul site de Bougouni. Ce site est situé en zone soudanienne sud caractérisé par un climat humide favorisant la survie et la multiplication du parasite et de ses vecteurs. Une étude réalisée par Deconinck et al. (1996) (8), a permis d'obtenir une prévalence très élevée chez les petits ruminants en Côte d'Ivoire (68\%), pays très humide, comparativement aux pays relativement plus secs ayant enregistré des taux inférieurs à $30 \%$.L'étude a permis d'établir une variation des taux de prévalence en fonction de l'espèce de petits ruminants $(7,06 \%$ chez les moutons et 2,57 chez les caprins). Ainsi, les ovins ont été plus fréquemment infectés par la toxoplasmose et la chlamydiose. Ceci a été confirmé par Deconinck et al. (1996) (8), qui a obtenu des prévalences plus élevées chez les ovins au Sénégal et en Ethiopie avec $11,5 \%$ et $25,6 \%$ respectivement contre $3,5 \%$ et $19,5 \%$ chez les caprins pour la toxoplasmose. Les taux de prévalence les plus élevés ont été obtenus en chlamydiose chez les animaux âgés de 1 à 4 ans $(\mathrm{p}=0,071>0,05)$, période de reproduction intense au cours de laquelle le maximum d'animaux s'infectent plus fréquemment. L'analyse des résultats en fonction de la nature de la perte en 
reproduction montre que sur l'ensemble des types de pertes en reproduction enregistrés au cours de l'étude, les avortements occupent une place de choix $(87,38 \%)$. Suite à la recherche d'anticorps spécifiques des différents agents des maladies abortives concernées dans 762 sérums issus d'animaux ayant avorté, 73 se sont révélés positifs. Les autres cas d'avortements (689) seraient probablement dus à d'autres facteurs, notamment d'autres maladies abortives comme la fièvre Q (15), certaines maladies infectieuses, en l'occurrence la pasteurellose, la peste des petits ruminants, la clavelée/variole caprine ou les cas de forte infestation par les parasites et l'état de carence nutritionnelle.

\section{Conclusion}

Cette étude a permis a permis de confirmer la circulation des agents étiologiques de la toxoplasmose (Toxoplasma gondii), de la chlamydiose (Chlamydia abortus) et de la brucellose à Brucella ovis chez les petits ruminants au Mali, les anticorps mis en évidence étant d'origine infectieuse (animaux non vaccinés contre les maladies investiguées). Les taux de séroprévalence ont varié en fonction du site de l'étude (taux d'infection en brucellose à Brucella ovis plus élevé à Koro, suivi de Bougouni, taux de séroprévalence en chlamydiose plus élevé à Nioro suivi de Ségou, cas de toxoplasmose enregistrés dans le seul site de Bougouni), du type de perte en reproduction (prédominance de cas positifs chez les animaux avorteurs), de l'espèce de petits ruminants (ovins plus fréquemment infectés par la toxoplasmose et la chlamydiose), de l'âge des animaux testés (taux de prévalence plus importants chez les sujets âgés de plus de 4 ans).

Conflit d'intérêt : Aucun

\section{Références}

1. Rapport de L'Institut National de la Statistique, Comptes économiques du Mali 2017

2. Rapport annuel de la Direction Nationale des Productions et Industries Animales (DNPIA), 2017.

3. A Souriau , C DE SA, A Rodolaxis. Chlamydiose abortive et vaccination, Renc Rech Ruminats. 3, 1996, 153- 156.

4. A Faye. Les Maladies de la reproduction chez les petits ruminants au Sénégal : étude sérologique de quatre infections bactériennes majeures (brucellose, chlamydiose, listériose, fièvre Q). Thèse de Doctorat d'Etat, Université Cheick Anta DIOP de Dakar. Ecole Inter-Etats des Sciences et Médecine Vétérinaires, 1992 No5.

5. Adamou A. Contribution à l'étude de la fièvre $\mathrm{Q}$ et de la chlamydiose bovine: enquête sérologiques dans la région du Nord Cameroun. Thèse Med. Vét. $\mathrm{N}^{\circ} 7$, Dakar, 1982 .

6. Akakpo A.J., Teou K.L, Kponmassi T., Zeller H. G. (avec la collaboration technique de Dieng M.et de Sene M.). Epidémiologie des affections abortives au Togo: enquête sérologiques sur la brucellose, la chlamydiose, la fièvre Q et la fièvre de la vallée du Rift. Biotechnologie $\mathrm{du}$ diagnostic et de la prévention des maladies animales, Ed. AUPELF-UREF, Jon Libbey Eurotext, 1994, P. 125-137.

7. Chartier C., Chartier F. Enquête séroépidémiologique sur les avortements infectieux des petits ruminants en Mauritanie. Revue Elev. Méd. Vêt. Pays Trop. 241 (1), 1988: p.23-34.

8. Deconinck P., Pangui L.J., Akakpo J., Garrouste A., Ouattara L., Roger F., et al. Prévalence de la toxoplasmose chez les petits ruminants en Afrique tropicale: résultats d'une enquête séroépidémiologique sur 1042 animaux, Revue de Médecine Vétérinaire, 147 (5) 1996: 377-378.

9. Jourdin E. Etude des maladies abortives non réglementées chez les ongulés 
sauvages et domestiques de la Réserve Nationale de Chasse et de Faune Sauvage des bauges. Thèse Lyon I, Université Claude Bernard. 2003, P. 41, 87-90.

10. Kibirige-Sebunya $T$. et Diteko. Diseases of small ruminants in Botswana 1983-92. Small Ruminants Research and Development in Africa. Proceedings of the Third Biennial Conference of the African Small Ruminant Research Network UICC, 1994, Kampala, Uganda.

11. Médina Khoumassi KABOU, A. Cherif, Salah Hammani, A. Majok.

Séroprévalence des maladies infectieuses majeures causant l'avortement chez les petits ruminants. In «Durabilité des systèmes d'élevage des petits ruminants en Tunisie : une approche de santé animale et Marketing », Discussion Paper N¹7, 2009 ILRI (International Livestock Research Institute.
12. Rekiki A., Thabti F., Dlissi I. et al., Enquête sérologique sur les principales causes d'avortements infectieux chez les petits ruminants en Tunisie, Revue Méd. Vétérinaire, 7, 2005;156: p.395-401.

13. Schelling E., Diguimbaye C., Daoud S., Nicolet et al.,. Séroprévalence des maladies zoonotiques chez les pasteurs nomades et leurs animaux dans le ChariBaguirmi au Tchad. Méd. Trop., 2004; 64: p. 474-7.

14. Rodolakis A., Armel S. et Berri M. Pathologie infectieuse et Immunologie, INRA, 2000

15. S.S. Sidibé, K.W. Coulibaly, M. Dakouo et al. Fièvre $Q$ chez les petits ruminants au Mali. Résultats d'une enquête sérologique. Revue d'élevage et de médecine vétérinaire des pays tropicaux, 2013, 66 (1) :11-18.

Tableau I. Prévalence par site des principales maladies abortives

\begin{tabular}{|c|c|c|c|c|c|c|c|}
\hline \multicolumn{8}{|c|}{ Sérums testés par pathologies } \\
\hline \multirow{2}{*}{$\begin{array}{c}\text { Sites } \\
\text { d'étude }\end{array}$} & \multirow{2}{*}{$\begin{array}{l}\text { Nbre total } \\
\text { sérums } \\
\text { testés }\end{array}$} & \multicolumn{2}{|c|}{ Chlamydiose } & \multicolumn{2}{|c|}{ Brucellose } & \multicolumn{2}{|c|}{ Toxoplasmose } \\
\hline & & $\begin{array}{l}\text { Nbre } \\
\text { Pos. }\end{array}$ & $\%$ & $\begin{array}{l}\text { Nbre } \\
\text { Pos. }\end{array}$ & $\%$ & $\begin{array}{l}\text { Nbre } \\
\text { Pos. }\end{array}$ & $\%$ \\
\hline Ségou & 306 & 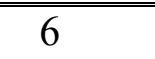 & 1,96 & 2 & 0,65 & 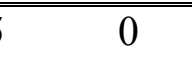 & 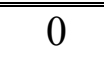 \\
\hline Nioro & 182 & 20 & 10,98 & 0 & 0 & 0 & 0 \\
\hline Bougouni & 205 & 4 & 1,95 & 3 & 1,46 & 39 & 19,02 \\
\hline Koro & 179 & 1 & 4,46 & 6 & 3,35 & 0 & 0 \\
\hline Total & 872 & 31 & 3,55 & 11 & 1,26 & 39 & 4,47 \\
\hline \multicolumn{4}{|l|}{ Variables } & \multicolumn{2}{|l|}{ khi-2 } & \multicolumn{2}{|l|}{ P-value } \\
\hline \multicolumn{4}{|c|}{ Global sites vs pathologies } & \multicolumn{2}{|l|}{252.25} & \multicolumn{2}{|l|}{$<2.2 \mathrm{e}-16$} \\
\hline \multicolumn{4}{|c|}{ Chlamydiose vs site } & \multicolumn{2}{|l|}{37.829} & \multicolumn{2}{|l|}{$3.073 \mathrm{e}-08$} \\
\hline \multicolumn{4}{|c|}{ Brucellose vs site } & \multicolumn{2}{|l|}{9.5805} & \multicolumn{2}{|l|}{0.02249} \\
\hline \multicolumn{4}{|c|}{ Toxoplasmose vs site } & \multicolumn{2}{|l|}{132.83} & \multicolumn{2}{|l|}{$<2.2 \mathrm{e}-16$} \\
\hline \multicolumn{4}{|c|}{ Ségou vs pathologie abortive } & \multicolumn{2}{|l|}{7.0615} & \multicolumn{2}{|l|}{0.02928} \\
\hline \multicolumn{4}{|c|}{ Niono vs pathologie abortive } & \multicolumn{2}{|l|}{41.521} & \multicolumn{2}{|l|}{$9.635 \mathrm{e}-10$} \\
\hline \multicolumn{4}{|c|}{ Bougouni vs pathologie abortive } & \multicolumn{2}{|l|}{59.258} & \multicolumn{2}{|l|}{$1.356 \mathrm{e}-13$} \\
\hline \multicolumn{4}{|c|}{ Koro vs pathologie abortive } & 8.9741 & & \multicolumn{2}{|l|}{0.01125} \\
\hline
\end{tabular}


Tableau II : Séroprévalence logiques en fonction de l'espèce de petits ruminants

\begin{tabular}{|c|c|c|c|c|c|c|c|}
\hline \multirow[t]{3}{*}{ Espèces } & \multirow{3}{*}{$\begin{array}{l}\text { Nombre } \\
\text { sérums } \\
\text { testés } \\
\end{array}$} & \multicolumn{6}{|c|}{ Sérums Positifs } \\
\hline & & \multicolumn{2}{|c|}{ Brucellose } & \multicolumn{2}{|c|}{ Chlamydiose } & \multicolumn{2}{|c|}{ Toxoplasmose } \\
\hline & & Nbre & $\%$ & Nbre & $\%$ & Nbre & $\%$ \\
\hline Ovine & 368 & 7 & 1,90 & 15 & 4,07 & 26 & 7,06 \\
\hline Caprine & 504 & 4 & 0,79 & 16 & 3,17 & 13 & 2,57 \\
\hline Total & 872 & 11 & 1,26 & 31 & 3,55 & 39 & 4,47 \\
\hline \multicolumn{4}{|c|}{ Variables } & \multicolumn{2}{|c|}{ khi-2 } & \multicolumn{2}{|c|}{ P-value } \\
\hline \multicolumn{4}{|c|}{ Global espèces vs pathologies } & \multicolumn{2}{|c|}{31.612} & \multicolumn{2}{|c|}{$7.091 \mathrm{e}-06$} \\
\hline \multicolumn{4}{|c|}{ Chlamydiose vs espèce animale } & \multicolumn{2}{|c|}{0.2755} & \multicolumn{2}{|l|}{0.5997} \\
\hline \multicolumn{4}{|c|}{ Brucellose vs espèce animale } & \multicolumn{2}{|c|}{1.3028} & \multicolumn{2}{|l|}{0.2537} \\
\hline \multicolumn{4}{|c|}{ Toxoplasmose vs espèce animale } & \multicolumn{2}{|c|}{8.9954} & \multicolumn{2}{|c|}{0.002707} \\
\hline \multicolumn{4}{|c|}{ Espèce ovine vs pathologie abortive } & \multicolumn{2}{|c|}{11.892} & \multicolumn{2}{|c|}{0.002616} \\
\hline \multicolumn{4}{|c|}{ Espèce caprine vs pathologie abortive } & \multicolumn{2}{|c|}{7.2491} & \multicolumn{2}{|c|}{0.02666} \\
\hline
\end{tabular}

Tableau III : Résultats par groupe d'âge des examens sérologiques

Sérums testés par pathologies

\begin{tabular}{|c|c|c|c|c|c|c|c|}
\hline \multirow{2}{*}{$\begin{array}{l}\text { Groupes } \\
\text { d'âge }\end{array}$} & \multirow{2}{*}{$\begin{array}{c}\text { Nbre total } \\
\text { sérums testés }\end{array}$} & \multicolumn{2}{|c|}{ Chlamydiose } & \multicolumn{2}{|c|}{ Brucellose } & \multicolumn{2}{|c|}{ Toxoplasmo-se } \\
\hline & & $\begin{array}{l}\text { Nbre } \\
\text { Pos. }\end{array}$ & $\%$ & $\begin{array}{l}\text { Nbre } \\
\text { Pos. }\end{array}$ & $\%$ & $\begin{array}{l}\text { Nbre } \\
\text { Pos. }\end{array}$ & $\%$ \\
\hline $0-1$ an & $\overline{107}$ & 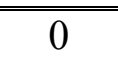 & 0 & 4 & 3,73 & 2 & 1,86 \\
\hline $1-4$ ans & 698 & 27 & 3,86 & 7 & 1 & 29 & 4,15 \\
\hline$>4$ ans & 67 & 4 & 5,97 & 0 & 0 & 8 & 11,94 \\
\hline Total & 872 & 31 & 3,55 & 11 & 1,26 & 39 & 4,47 \\
\hline \multicolumn{4}{|l|}{ Variables } & khi-2 & \multicolumn{3}{|c|}{ P-value } \\
\hline \multicolumn{4}{|c|}{ Global tranches d'âge vs pathologies abortives } & 39.741 & \multicolumn{3}{|c|}{$3.58 \mathrm{e}-06$} \\
\hline \multicolumn{4}{|c|}{ Chlamydiose vs tranches d'âge } & 5.2835 & \multicolumn{3}{|c|}{0.07124} \\
\hline \multicolumn{4}{|c|}{ Brucellose vs tranches d'âge } & 6.5009 & \multicolumn{3}{|c|}{0.03876} \\
\hline \multicolumn{4}{|c|}{ Toxoplasmose vs tranches d'âge } & 10.608 & \multicolumn{3}{|c|}{0.004972} \\
\hline \multicolumn{4}{|c|}{ Tranche d'âge (0-1an) vs pathologies abortives } & 4.0762 & \multicolumn{3}{|c|}{0.1303} \\
\hline \multicolumn{4}{|c|}{ Tranche d'âge (1-4ans) vs pathologies abortives } & 14.532 & \multicolumn{3}{|c|}{0.0006987} \\
\hline \multicolumn{4}{|c|}{ Tranche d'âge ( $>$ 4ans) vs pathologies abortives } & 8.5079 & \multicolumn{3}{|c|}{0.01421} \\
\hline
\end{tabular}


Tableau IV: Prévalence en fonction de la nature de la perte en reproduction

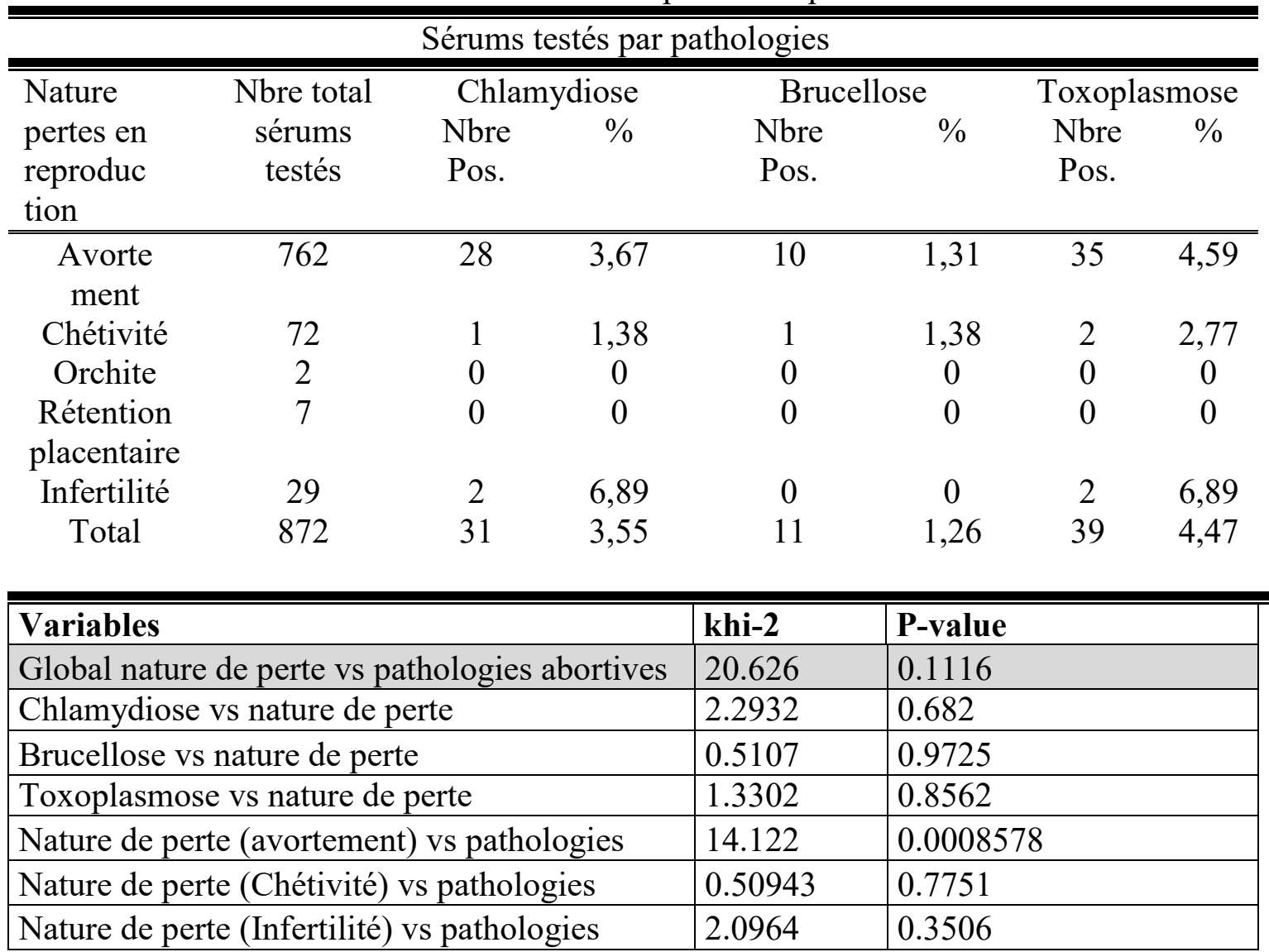

